\title{
Final focus system for injection into a laser plasma accelerator
}

\author{
S. Yamin $\odot,{ }^{* \dagger}$ R. Assmann, F. Burkart, A. Ferran Pousa®, F. Lemery, and E. Panofski \\ DESY, Notkestrasse 85, 22607 Hamburg, Germany \\ W. Hillert \\ University of Hamburg, 20148, Hamburg, Germany \\ B. Marchetti \\ European XFEL GmbH, Holzkoppel 4, 22869 Schenefeld, Germany
}

(Received 18 February 2021; accepted 17 August 2021; published 15 September 2021)

\begin{abstract}
ARES is an electron linear accelerator at the SINBAD facility at DESY. It aims to deliver reliable highbrightness beams with an energy in the range of 100 to $150 \mathrm{MeV}$ with fs to sub-fs bunch lengths. This is ideal for injection into novel high-gradient acceleration devices, such as dielectric laser accelerators and laser-plasma accelerators (LPAs), which feature fields with fs to ps period. Here, we report the conceptual design of a final focus system for injecting into an LPA experiment at ARES. The design includes permanent magnetic quadrupoles (PMQ), sufficient longitudinal space for collinear laser and electron transport, space for required diagnostics and an LPA setup. The performance of the design is evaluated by means of start-to-end simulations of the linac, focusing system, and injection into the LPA, including sensitivity studies to relevant error sources.
\end{abstract}

DOI: 10.1103/PhysRevAccelBeams.24.091602

\section{INTRODUCTION}

Short Innovative Bunches and Accelerators at DESY (SINBAD) is an accelerator R\&D facility at DESY on its Hamburg site [1]. It includes research in the field of ultrashort electron bunches and will host multiple independent experiments [2] on laser-driven advanced highgradient acceleration techniques such as dielectric laser acceleration (DLA) [3], laser plasma acceleration (LPA) [4] and THz-driven acceleration in the AXSIS project [5]. The ARES (Accelerator Research Experiment at SINBAD) linear accelerator (linac) at SINBAD is based on conventional S-band technology with a photoinjector gun [6,7]. It is designed to provide ultrashort, high-brightness electron beams for injection into novel accelerators. The FWHM duration of electron bunches is expected to reach a few-fs and potentially sub-fs values [8]. The electron gun relies on a conventional radio frequency (rf) accelerator technology for producing the electron bunches which has several advantages. The ARES linac on one hand will allow advancing $\mathrm{R} \& \mathrm{D}$ on the "conventional" production of

\footnotetext{
*sumera.yamin@desy.de

Also at University of Hamburg, 20148, Hamburg, Germany.
}

Published by the American Physical Society under the terms of the Creative Commons Attribution 4.0 International license. Further distribution of this work must maintain attribution to the author(s) and the published article's title, journal citation, and DOI. high-brightness ultrashort electron bunches. On the other hand, the well-characterized bunches can be used to explore compact novel accelerators, characterized by accelerating fields with short wavelengths and therefore require the injection of short bunches. The ARES bunches have been designed to constitute excellent probes to measure the energy gain and the quality of the acceleration.

At ARES, the electron bunches can be compressed either via velocity bunching, by using a magnetic bunch compressor or by using a hybrid scheme to achieve the desired bunch lengths in the range of sub-fs to few fs $[8,9]$. The characterization of such ultrashort bunches is a research field in itself and ARES will also serve as a test bench for novel diagnostic devices in the low to medium charge range of up to $30 \mathrm{pC}$ and with sub-fs to few-fs bunch length $[10,11]$. All of these features contribute in making ARES a promising candidate where LPA experiments with external injection could be performed.

In an LPA, an intense laser pulse propagates in a plasma and generates charge separation through the excitation of wakefields, inducing strong electric fields. LPAs can provide an accelerating gradient in the range of $\sim 100 \mathrm{GV} / \mathrm{m}$, which is several orders of magnitude higher than what can be achieved with conventional rf technology. This reduces the size of the acceleration channel to millimeter scales, to achieve $\mathrm{GeV}$ energies, enabling the development of compact and cost-effective accelerators. Since the LPA concept was first introduced [12], it has been a subject of extensive studies with significant progress in 
recent years [13,14]. Many important milestones have been demonstrated such as achieving $\mathrm{GeV}$ energies in only $\mathrm{cm}$ scales [15]. Today's focus for these devices is to reach beam stability, similar to established rf accelerators, by deploying feedback control systems. Recently another milestone has been successfully demonstrated at DESY with 24 hours of stable operation of laser plasma acceleration [16].

The external injection LPA experiment studied for ARES investigated a possible step towards usable LPA from a combination of reproducible conventional rf-based accelerator technology with the high gradient fields from plasma wakefields [17]. The rf-based technology allows for a precise manipulation of the phase space of the electron bunches before entering the plasma hence, providing independent control and quality adjustments as well as optimization of the plasma experiment. The beam quality in novel accelerators depends on the detailed parameters and quality of the injected beam, e.g. bunch shape, bunch length, emittance, arrival time stability and beam energy. ARES provides the option of widely tunable working points (WPs) and bunch shapes for external injection LPA accelerator R\&D. External injection of electron beams into an LPA, however, has its own technical challenges [7]. The synchronization of laser and electron beam can be the most crucial aspect of this experiment.

The present status of ARES is shortly summarized. Its $5 \mathrm{MeV}$ rf gun and linac has been commissioned [18] and first electrons have been produced at the end of 2019 [19]. The installation of the subsequent experimental chamber and diagnostic beam line is finished [19]. The preparations for a DLA experiment are ongoing and simulation studies have been performed for a potential LPA experiment [20]. In this paper, we present design studies for a final focus system that could fulfill the requirements of external injection of ARES bunches into an LPA setup. In the following sections, the layout of the ARES linac and the experimental area for the simulated LPA experiment are described, followed by the requirements for beam matching into the plasma cell. The results for design and optimization of the final focus system and for electron beam tracking through the lattice are presented. Tolerance studies for the final focus system are presented and discussed. The aim of this article is to present the design of the final focus system which fulfills the matching criterion for the LPA experiment within the defined constraints. Plasma simulations presented validate the design studies. The design constraints are in general typical to any LPA experiment with external injection of electron beam. The matching criterion depends mostly upon the laser and plasma parameters. The presented design studies for the final focus system and the error analysis can be generalized and adopted for an LPA experiment with external injection of electron beam.

\section{LAYOUT OF ARES LINAC}

A schematic overview of the ARES linac with a potential LPA acceleration experiment is shown in Fig. 1. The main beam parameters are summarized in Table I. The ARES linac consists of a $5 \mathrm{MeV}$ rf gun followed by two traveling wave structures with space reserved for a third traveling wave structure for a possible future energy upgrade [7]. This space will be temporarily used as a first experimental area. This is followed by a matching section into a magnetic bunch compressor (BC). At the exit of the $\mathrm{BC}$ the electron bunches have a duration of a few fs or below [21]. The BC is followed by a drift space that provides space for the laser in-coupling. Further downstream, we have a high energy diagnostic beam line followed by the matching optics for the second experimental area. This area could host LPA experiments and could combine the electron beam from ARES with the Joule-class laser pulses from the high repetition rate laser, KALDERA, currently under

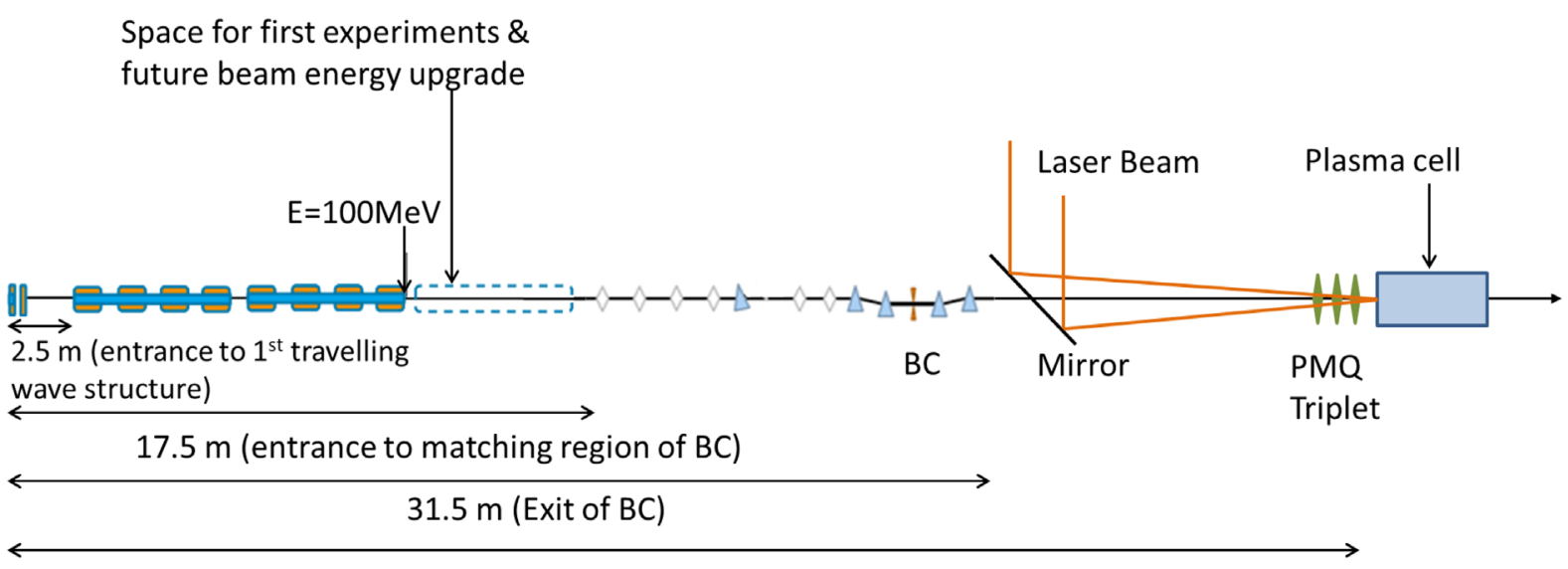

$35 \mathrm{~m}$ (Entrance of Plasma Cell)

$\checkmark$ Quadrupoles $\triangle$ Dipoles $\quad|l|$ Quadrupole Triplet

FIG. 1. Schematic of the ARES beam line including injection into an LPA (not to scale). 
TABLE I. Parameters for the rf systems and the electron beam at the ARES linac.

\begin{tabular}{lc}
\hline \hline Parameter & Values \\
\hline rf frequency & $2.998 \mathrm{GHz}$ \\
Repetition rate & $50 \mathrm{~Hz}$ \\
Beam energy & $100 \mathrm{MeV}(155 \mathrm{MeV}$ on crest $)$ \\
Upgraded beam energy & $150 \mathrm{MeV}(230 \mathrm{MeV}$ on crest $)$ \\
Bunch charge & $0.5-30 \mathrm{pC}$ \\
Bunch length & sub to few fs \\
Arrival time jitter stability & 10 fs to few tens of fs \\
\hline \hline
\end{tabular}

development at DESY [22]. As mentioned in Sec. I, ARES is designed to have widely tunable beam parameters over the range given in Table I. A number of working points (WPs), machine settings delivering beam with specified parameters, have been established for ARES [23]. Beam parameters for each WP of ARES corresponds to different applications. The matching scheme designed for the low charge WP of the ARES linac, which will provide $0.8 \mathrm{pC}$ electron bunches with smallest arrival time jitter of about $\sim 10 \mathrm{fs}$ rms, has been discussed in detail in [24]. It uses a pure magnetic compression scheme and is ultimately designed for sub-fs bunch lengths. The electron bunches from this setup provide a time resolution in the sub-fs range and can serve as probe particles for plasma wakefields or fields in dielectric structures.

In this paper, we present the results for the higher charge $10 \mathrm{pC}$ WP of the ARES linac. It features a longer bunch length of $10 \mathrm{fs}$ FWHM or $4.3 \mathrm{fs}$ rms. The peak current in this case is $1 \mathrm{kA}$, approaching the requirements of several use cases [25]. It is noted that this WP is similar to a WP studied at the BELLA facility in LBNL for which a broad energy spread electron bunch of $\sim 100-200 \mathrm{MeV}$ energy, $6.25 \mathrm{pC}$ charge and $3.3 \mathrm{fs}$ rms length was used for injection into a second stage LPA [26].

\section{TECHNICAL CONSTRAINTS FOR THE MATCHING BEAM LINE}

In the external injection LPA experiment, the laser and electron beams are collinearly injected into the plasma target. This collinearity, together with the required beam diagnostic elements, introduces strict constraints on both the transverse and longitudinal dimensions of the final focus system. On one side, the tight matching requirements for the electron beam into the plasma (cf. Sec. III) imply the need for large focusing gradients and, hence, tight apertures. On the other hand, the longitudinal extent of the system is constrained due the tight apertures and the laser beam size, which for a Gaussian pulse is given by [27]

$$
w(z)=w_{0} \sqrt{1+\left(\frac{z}{Z_{R}}\right)^{2}},
$$

TABLE II. Key parameters for the laser setup.

\begin{tabular}{lcc}
\hline \hline Parameters & Units & Values \\
\hline Wavelength $\left(\lambda_{0}\right)$ & $\mu \mathrm{m}$ & 0.8 \\
Peak normalized vector potential $\left(\mathrm{a}_{0}\right)$ & & 1.4 \\
Beam waist $\left(w_{0}\right)$ & $\mu \mathrm{m}$ & 40 \\
Pulse energy $(\mathrm{E})$ & $\mathrm{J}$ & 3 \\
Peak power $(\mathrm{P})$ & $\mathrm{TW}$ & $\sim 100$ \\
Pulse length $\left(\Delta t_{\mathrm{FWHM}}\right)$ & $\mathrm{fs}$ & 30 \\
\hline \hline
\end{tabular}

where $Z_{R}=\pi w_{0}^{2} / \lambda_{0}, w_{0}$ and $\lambda_{0}$ are, respectively, the Rayleigh length, spot size at focus and wavelength of the laser, while $z$ represents the distance to its focal position.

A promising option for fulfilling these requirements is the use of a Halbach-type permanent magnetic quadrupole (PMQ) triplet [28]. This offers large focusing gradients in excess of $100 \mathrm{~T} \mathrm{~m}^{-1}$ with only $\sim 1 \mathrm{~cm}$ long magnets. The use of this technology has already been explored for applications such as inverse Compton scattering [29] as well as for the focusing, capture and transport of beams from plasma accelerators [30-34]. As a result, a PMQ triplet has also been chosen for the final focus system at ARES.

The key parameters for the laser system are presented in Table II. The schematic of the laser beam at its waist and the parameter definitions are shown in Fig. 2. For this study a Gaussian laser beam is assumed. Since, as given by Eq. (1), the divergence of the laser beam depends on $w_{0}$, the design of the laser beam line and the space constraints for our final focus system are strongly dependent on the laser parameters.

The technical design considerations for the PMQ triplet have been discussed in detail in [35]. The required mirror for the laser in-coupling is housed in a beam pipe with a diameter of $10 \mathrm{~cm}$. The beam pipe size is chosen to accommodate the mirror dimensions required for focusing the $100 \mathrm{TW}$ peak power laser beam. A hole in the mirror allows the electron beam transmission and, collinearly to the laser beam, the electron beam enters the plasma cell. At the focal point, the laser beam has a design waist of $40 \mu \mathrm{m}$. For the external injection experiment, the laser beam also

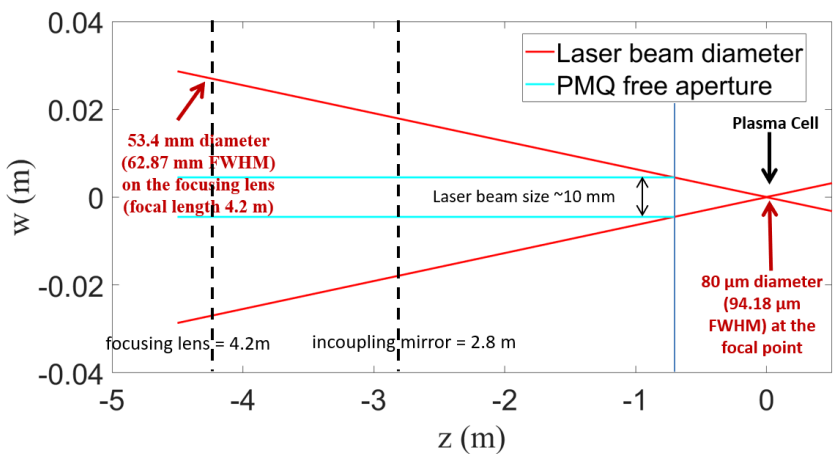

FIG. 2. Evolution of the laser beam along the direction of beam propagation. The origin here is set at the focal point of the laser beam which is the entrance position of the plasma cell. A Gaussian laser beam is assumed. 
has to pass through the PMQ triplet to reach the plasma cell. Hence the free aperture, defined by the distance of the magnetic pole tips of the PMQ, should be bigger than the laser spot size at the position of the quadrupoles. Considering the laser beam evolution from Fig. 2, the aperture of PMQ is chosen to be $10 \mathrm{~mm}$. The laser parameters and the laser beam line design dictate the limits for the physical dimensions of the PMQ inner and outer radii, the length of the total triplet and also set constraints to the positions of the focusing magnet. The distance between the exit of the $\mathrm{BC}$ and the entrance of the plasma cell is $\sim 3.5 \mathrm{~m}$ to account for the space required for diagnostics, the vacuum system and for focusing the laser beam.

\section{MATCHING CRITERION}

The transverse properties of an electron bunch are defined by emittance $\epsilon$ and the Courant-Snyder parameters $\beta, \alpha$ and $\gamma$, also often referred to as Twiss parameters.

Emittance and Twiss parameters are related to shape of the beam ellipse. Emittance is defined as area occupied by beam in phase space. $\beta_{x}$ is related to beam size in $x, \gamma_{x}$ is related to beam size in $x^{\prime}$ and $\alpha_{x}$ is related to tilt angle of ellipse $x x^{\prime}$, where $x^{\prime}=p_{x} / p_{z}$ is the slope of particle trajectory. Twiss parameters are related as $\gamma=\left(1+\alpha^{2}\right) / \beta$ [36]. In this paper, Twiss parameters $\beta$ and $\alpha$ have been used to define the matching criterion.

In order to preserve the beam emittance during acceleration in the LPA, matching the Twiss parameters of the injected electron beam to those determined by the large focusing fields inside the plasma is required [37]. Assuming $\alpha_{x, y}=0$, the matched value of the beta function, for a plasma stage with a uniform focusing gradient $g$, is given by $[37,38]$

$$
\beta_{m}=\sqrt{\frac{c m_{e}}{e} \frac{\gamma_{r}}{g}}
$$

where $g$ is the focusing gradient (in units of $\mathrm{T} \mathrm{m}^{-1}$ ) inside the LPA, $\gamma_{r}$ is the relativistic Lorentz factor of the beam and $e$ is the electron charge.

In the LPA stage considered here, which features a plasma electron density of $10^{17} \mathrm{~cm}^{-3}$ and a laser driver with the parameters from Table II, the resulting focusing gradient is $g \simeq 1.1 \mathrm{MT} \mathrm{m}^{-1}$ at the center of the stage. Thus, for a beam energy of $150 \mathrm{MeV}$, Eq. (2) yields $\beta_{m} \simeq 0.7 \mathrm{~mm}$. This is a strong requirement that could be challenging to achieve, even with the considered PMQ triplet. Fortunately, this condition can be relaxed by using a properly tailored vacuum-toplasma transition (or upramp) [39-42]. In this study, a plasma upramp following the profile,

$$
n_{p, r}\left(z_{r}\right)=\frac{n_{p, 0}}{\left(1+\frac{z_{r}}{L_{r}}\right)^{2}}
$$

has been used, where $n_{p, r}$ is the plasma density along the ramp, $n_{p, 0}$ is the on-axis plasma electron density in the plateau, $z_{r}$ is the distance to the plateau, and $L_{r}$ is a parameter controlling the plasma density decay rate in the ramp. This profile has been shown to efficiently relax the matching requirements at the plasma entrance [41]. For the present case, $L_{r}=2 \mathrm{~mm}$ has been considered, with a total ramp length of $3 \mathrm{~cm}$. The resulting density profile can be seen in Fig. 8(a).

The required Twiss parameters at the entrance of such a ramp can be analytically calculated when the LPA operates in the so-called blowout regime [41], where a laser driver with $a_{0}^{2} \gg 1$ is able to generate a trailing ion column fully cavitated of plasma electrons, where $a_{0}$ is the peak normalized vector potential at focus [43]. This, however, given the laser parameters in Table II, is not the case here. As such, these parameters have been determined by means of tracking simulations with the WAKE-T code [44] using a nonlinear 1D fluid model for the plasma wakefields. This model, although simple, allows for an accurate estimate of the required beam parameters, as demonstrated by the results in Sec. VII. In the simulations, a particle bunch with the ARES beam properties, but with $\alpha_{x, y}=0$ and $\beta_{x, y}=\beta_{m}$, has been backpropagated from the entrance of the plateau until the beginning of the plasma upramp. This technique, also used in [40], yields a final beam with the required Twiss parameters at the ramp entrance such that a matched beam is achieved at the plateau. For the case simulated here, this means a beam with $\beta_{x, y}=$ $11.8 \mathrm{~cm}$ and $\alpha_{x, y}=4.4$, which are therefore the target Twiss parameters of the final focus system. These parameters are equivalent to a virtual focus with $\beta_{x, y} \simeq 5.8 \mathrm{~mm}$ at waist, a significant improvement over a case with no ramp.

\section{DESIGN STUDY FOR THE MATCHING BEAM LINE}

Start-to-end simulations were carried with a combination of ASTRA [45], ELEGANT [46] and IMPACT-T [47]. A total of

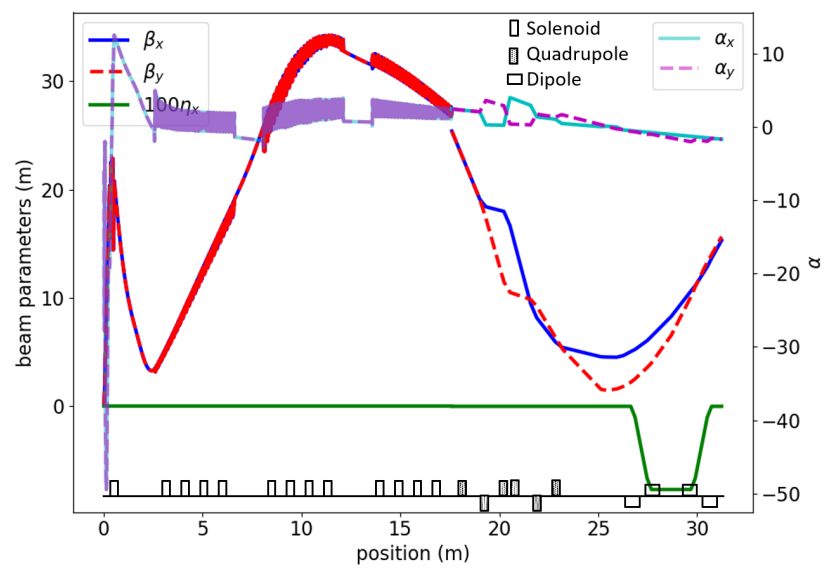

FIG. 3. The Twiss parameter $\beta_{x, y}$ and $\alpha_{x, y}$ and dispersion $\eta_{x}$ evolution is illustrated along the length of the linac. The BC terminates at $z \sim 31.5 \mathrm{~m}$, the plasma is anticipated to be located at $z \sim 35 \mathrm{~m}$. 


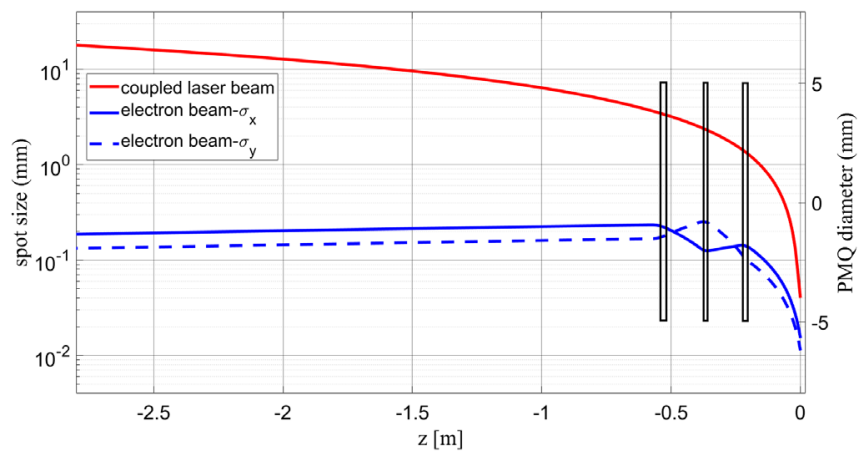

FIG. 4. Laser and electron beam propagation through the PMQ triplet. The laser and electron beam is plotted from the position of laser in-coupling mirror ( $2.8 \mathrm{~m}$ behind the plasma cell) until the entrance of the plasma cell. To the scale dimension and position of optimized quadrupole triplet are also shown.

four million macroparticles were used before the energy selective slit collimator in the bunch compressor. The rms beam size of the inverse parabolic laser shape on the cathode was 181 microns, while the Gaussian rms pulse duration was 3 ps, consistent with ARES laser infrastructure. IMPACT-T supported the calculation of coherent synchrotron radiation effects in the BC. Further information can be found in [21]. The Twiss parameters for start-to-end simulation until BC exit is shown in Fig. 3.

The beam dynamics simulations for the matching beam line after the $\mathrm{BC}$ have been performed using ELEGANT (without space charge) and then ASTRA to optimize and include the effects of 3D space charge (SC). The optimization parameters for the PMQ triplet are the lengths, strengths and distances between the quadrupoles. Based on the laser beam line layout, as shown in Fig. 2, the constraints for the final focus system were the quadrupole aperture, the outer diameter corresponding to size of the quadrupoles, and the focal length. The focal length must take into account space for diagnostic screens allowing laser and electron beam profiling between the last magnet of the triplet and the plasma cell. The optimizer scans the input parameters within the constraints defined in Sec. III to find a viable configuration for the quadrupole triplet. We set the origin of our simulations at the exit of the last magnet of the $\mathrm{BC}$ and the beam line is simulated from this point until the entrance of the plasma cell corresponding to the start of plasma upramp as defined by the matching criterion in Sec. IV.

The key parameters for the working points at the $\mathrm{BC}$ exit and at the entrance of the plasma cell, simulated in ASTRA, are summarized in Table III. The full beam distribution is included in the calculation of statistical parameters. The three quadrupoles of the triplet have lengths of $0.055,0.033$ and $0.040 \mathrm{~m}$ with strengths of $50,-180$ and $130 \mathrm{~m}^{-2}$ respectively. The distance between the last magnet and the plasma cell is $0.25 \mathrm{~m}$ which is sufficient to place diagnostics for laser and electron beam profiling as shown in Fig. 4. It is planned to adopt the interaction chamber for positioning the PMQ triplet, electron and laser beam diagnostic before and after the plasma cell as already implemented at REGAE, DESY [48]. Figure 5 shows the evolution of the electron beam parameters over the drift space and through the PMQ triplet from the $\mathrm{BC}$ to the plasma cell. The transverse and longitudinal phase spaces

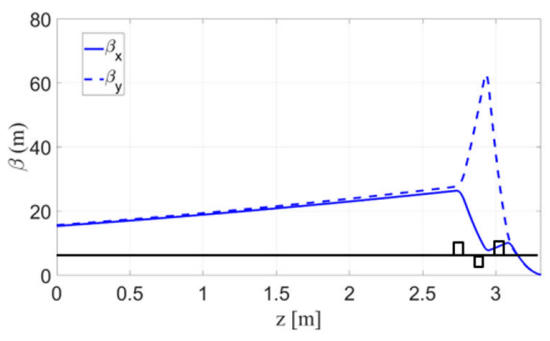

(a)

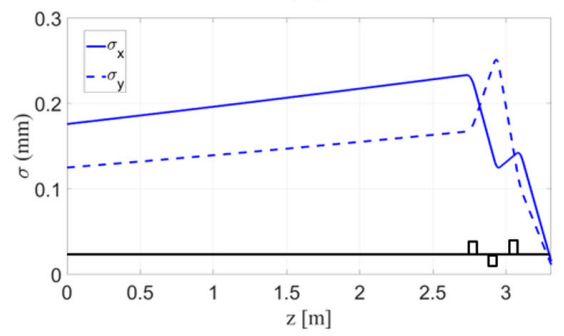

(d)

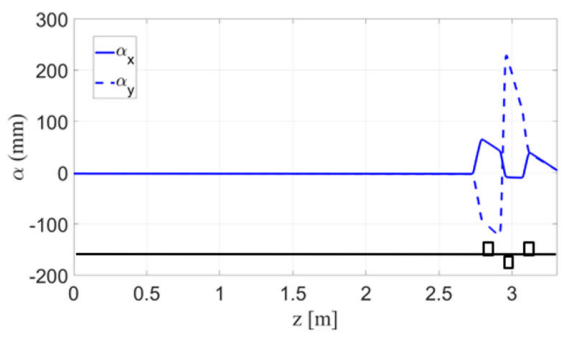

(b)

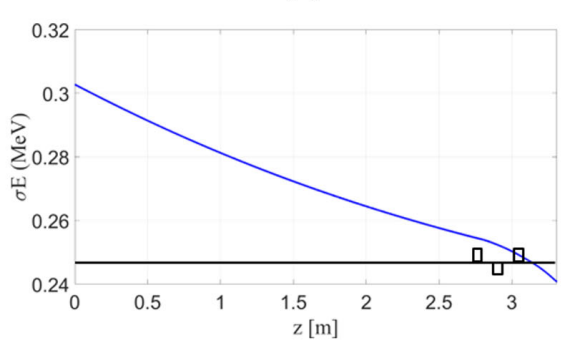

(e)

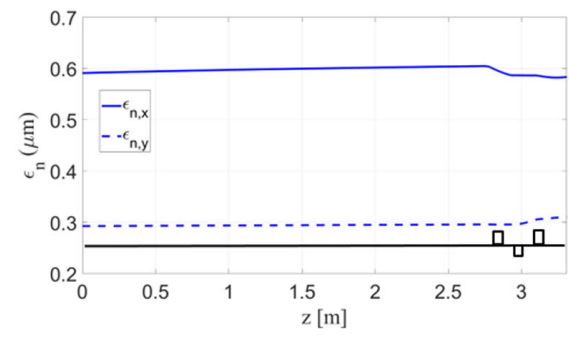

(c)

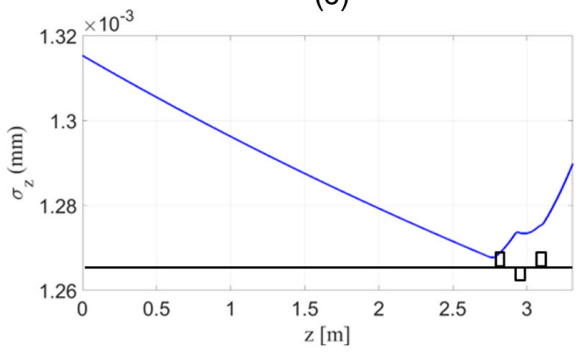

(f)

FIG. 5. Evolution of the electron beam parameters, simulated in ASTRA, along the beam line from the exit of the BC until the entrance of the plasma cell. The origin here is set at the exit of the last magnet of the BC. The parameters shown are the Twiss parameters in (a) $\beta_{x, y}$ and in (b) $\alpha_{x, y}$, in (c) the normalized transverse emittance $\varepsilon_{x, y}$, in (d) the rms transverse beam sizes $\sigma_{x, y}$, in (e) the energy spread $\sigma_{E}$ and in (f) the bunch length $\sigma_{z}$. 

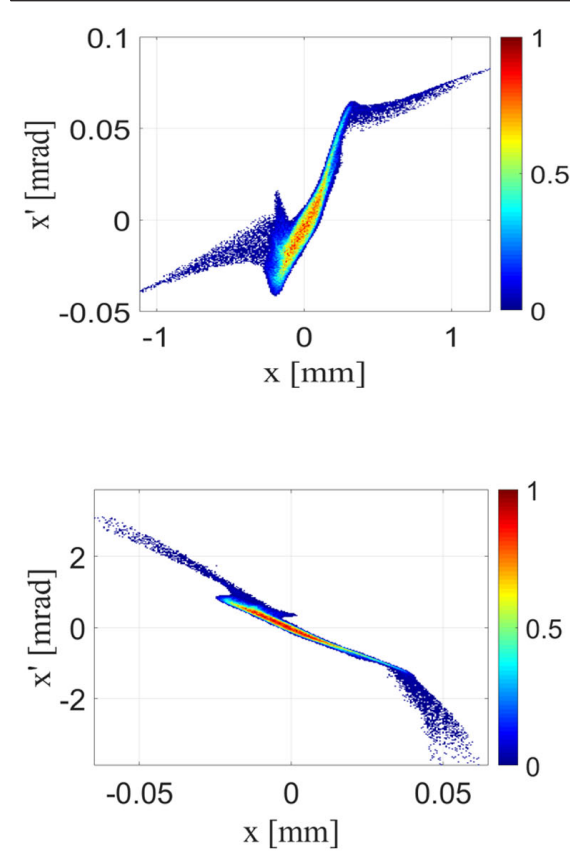

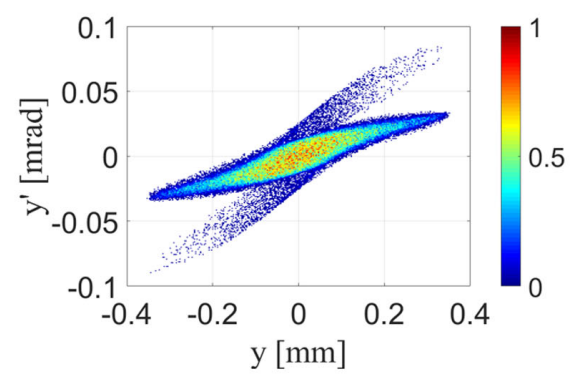

(a)

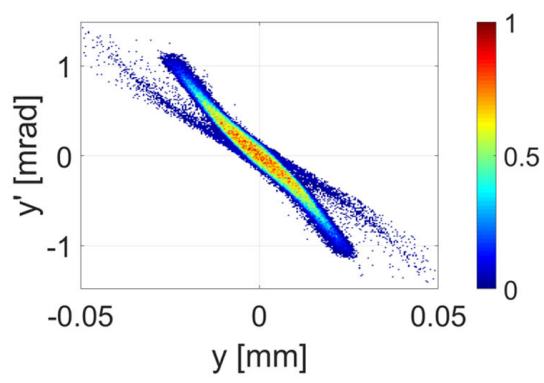

(b)
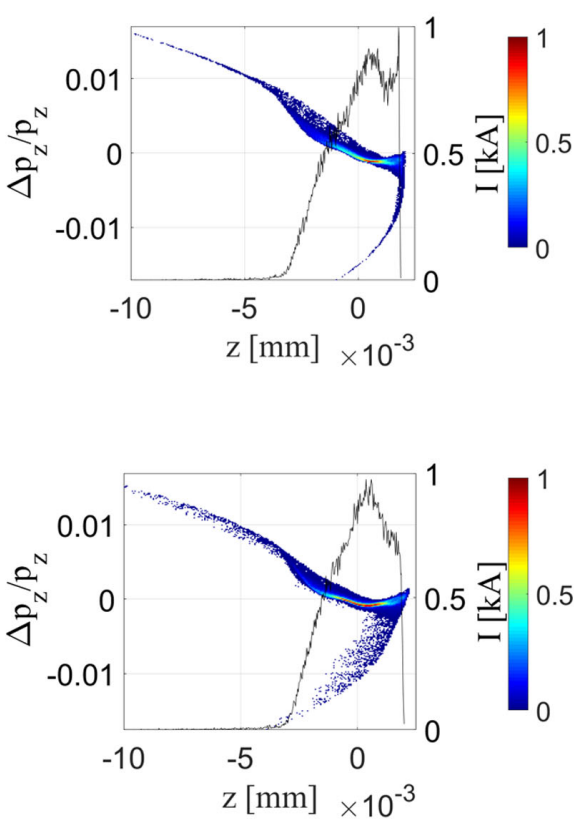

FIG. 6. Evolution of the transverse and longitudinal phase spaces, simulated in ASTRA, at (a) the BC exit and matched beam at (b) the entrance of the plasma cell. Color scales indicate normalized electron density.

of the electron beam at the $\mathrm{BC}$ exit and at the entrance of plasma cell are shown in Fig. 6. From Figs. 5 and 6 it is evident that at the start of plasma ramp, the electron beam is well matched with preserved transverse emittance and bunch length shorter than the accelerating wavelength in the plasma. The transverse phase space distributions show that the beam is symmetric in both planes as required by LPA. The PMQ triplet also ensures to maintain the current

TABLE III. Bunch parameters at the BC exit (I), matched beam at the entrance of plasma cell (II), accelerated beam after the plasma cell (III) and the matched beam at the plasma without including the effects of SC (IV). The same quadrupole settings are used for II and IV.

\begin{tabular}{lcccc}
\hline \hline Parameters & I & II & III & IV \\
\hline Energy (MeV) & 150 & 150 & 1000 & 150 \\
Bunch charge (pC) & 10 & 10 & 10 & 10 \\
Bunch length FWHM (fs) & 11.8 & 11.9 & 11.7 & 11.79 \\
Bunch length rms (fs) & 4.38 & 4.30 & 4.48 & 4.26 \\
$\varepsilon_{n, x}(\mu \mathrm{m})$ & 0.59 & 0.57 & 0.63 & 0.55 \\
$\varepsilon_{n, y}(\mu \mathrm{m})$ & 0.29 & 0.31 & 0.52 & 0.31 \\
$\beta_{x}(\mathrm{~cm})$ & $15.32 \times 10^{2}$ & 11.8 & 14.9 & 11.41 \\
$\beta_{y}(\mathrm{~cm})$ & $15.66 \times 10^{2}$ & 11.9 & 14.7 & 9.92 \\
$\alpha_{x}$ & -1.7 & 4.5 & -4.7 & 4.54 \\
$\alpha_{y}$ & -1.7 & 4.4 & -4.7 & 3.93 \\
$\sigma_{x}(\mu \mathrm{m})$ & 175.8 & 15.2 & 6.61 & 14.7 \\
$\sigma_{y}(\mu \mathrm{m})$ & 124.9 & 11.2 & 5.96 & 10.2 \\
rms energy spread $(\%)$ & 0.2 & 0.16 & 0.68 & 0.20 \\
Peak current $(\mathrm{kA})$ & 1 & 1 & 0.92 & 0.67 \\
\hline \hline
\end{tabular}

profile from the longitudinal phase space with $\sim 1 \mathrm{kA}$ of peak current.

It is worthwhile to note that SC impacts the beam parameters at $150 \mathrm{MeV}$ especially after the $\mathrm{BC}$ when the beam is compressed. Using the same quadrupole triplet settings, without SC, beam parameters at the entrance of the plasma cell are given in Table III. The beam parameters like $\beta_{x}=11.41 \mathrm{~cm}, \beta_{y}=9.92 \mathrm{~cm}, \alpha_{x}=4.54, \alpha_{y}=3.93 \sigma_{x}=$ $14.7 \mu \mathrm{m}$ and $\sigma_{y}=10.2 \mu \mathrm{m}$ differ significantly from the matched case. The beam is mismatched and can lead to undesired emittance growth during acceleration in the plasma cell. Therefore, it is important to include the impact of SC while tracking the beam through the lattice.

Detailed simulation studies on beam tracking in the experimental beam line through the PMQ triplet show that the optimized settings for the PMQ triplet provide a good quality working point. The electron beam can be transported and matched into the plasma cell with the PMQ triplet while preserving the beam quality. The transverse and longitudinal phase space distributions show good beam profiles for injecting into LPA. The symmetric beam distributions with a peak current of $1 \mathrm{kA}$ would be sufficient for efficient beam loading [49-51] in the plasma cell.

\section{ERROR ANALYSIS OF THE PMQ TRIPLET AND THE INCOMING BEAM}

The experimental conditions may vary from the ideal design parameters discussed in the previous sections due to temporal and spatial jitter in the beam. Moreover, there are 
additional sources of errors in the quadrupoles, arising during the manufacturing and installation of the final focus system. For example, offsets between the magnetic field center of the quadrupoles and the ideal beam line can lead to emittance growth and betatron oscillations in the plasma. A basic tolerance study for the final focus system was performed to estimate the effects of several error sources.

We consider two sources of errors in our system. One set of errors can arise from spatial and temporal jitter in the electron beam. The other set of errors is introduced from positioning errors in the quadrupole triplet. In simulation parameters in the beam distribution after the bunch compressor were varied and analyzed. In addition, error from transverse misalignment $\mathrm{x}_{\text {off }}$ and $\mathrm{y}_{\text {off }}$ in the quadrupole triplets, from rotation errors $x_{\text {rot }}$ in the $x-z$ plane, from rotation errors $y_{\text {rot }}$ in the $y-z$ plane, from rotation errors $z_{\text {rot }}$ in the $x-y$ plane, from longitudinal offset errors $z_{\text {off }}$ and from errors in the focusing strength $\mathrm{K}$ were included. The quadrupole parameters under study were varied within the given tolerances range. All quadrupoles were assigned the same error as one would realistically expect if errors arise from a common support girder and positioning system. For example, mounting errors between single elements on a common girder are usually much better corrected than offsets or drifts of the whole girder in the tunnel.

For the tolerance studies of jitter in the input beam, the beam properties were perturbed. Included were transverse offsets in electron bunch position in both transverse planes ( $x$ and $y$ ), errors in bunch size in both transverse planes, the beam momentum and divergence, bunch charge, bunch length as well as longitudinal offset in $\mathrm{z}$, all defined at the exit of the bunch compressor. It is important to consider variations in beam properties as this can be an outcome of all possible sources of error in the accelerator. The misalignment of the beam line element up to the $\mathrm{BC}$ can also give rise to additional kick in the beam. Nevertheless, Twiss parameters of the beam at the entrance of the PMQ triplet can be finely adjusted by six quadrupole magnets upstream of the chicane as shown in Fig. 1.

The observables in simulation include transverse normalized emittance, transverse beam sizes, beam divergence in both planes, Twiss parameters, bunch length and energy spread at the entrance of the plasma cell.

In order to assess the robustness of the PMQ triplet solution, 10,000 cases were simulated for two cases.

Mismatch case 1: Errors are randomly assigned to PMQ triplet and bunch parameters according to normal distribution with mean being the design parameter given in Table III. The assigned tolerance corresponds to rms width of the distribution. This includes errors in bunch position at the BC exit, beam size, bunch length and bunch charge. For the quadrupole triplet, transverse, rotational and longitudinal offset and quadrupole strength were varied. The minimum tolerance ranges given in Table III are reasonable expectation as it is according to the precision range of hexapods which can be used for the positioning of the quadrupole triplet [52].

Mismatch case 2: A same set of simulations was carried out in which the tolerance range for transverse and rotational offset of quadrupole was $100 \mu \mathrm{m}$ and $100 \mu \mathrm{rad}$ respectively.

The position for observing the final beam parameters in both cases was fixed at the matched case position of Table III, which is the entrance of plasma cell as explained in Sec. IV. Figure 7 summarizes the simulated beam parameters at the plasma entrance, as obtained in the two error cases. From Fig. 7, it can be concluded that the mismatch case 1 is an acceptable scenario, since the beam with this set of variations is still well matched with only a small emittance growth when it enters the plasma cell. It is worthwhile to note again that this also includes the variation in input beam parameters. The most critical source of error is the quadrupole strength and longitudinal position and hence has the strictest tolerances as shown in Table IV. It can be safely inferred that the PMQ triplet design is robust and can be used for matching the electron beam to the plasma channel under less than ideal experimental conditions as well.

Additional errors in the quadrupole triplet can arise during the manufacturing of the permanent magnets. Variations can occur from many sources such as the precision with which the magnetic blocks comprising them are constructed, both in geometry of the cutting of the blocks and in the magnitude and direction of their magnetic moments, as well as the geometrical constraints required to carefully assemble these blocks into a Halbach configuration. The peak gradient is difficult to precisely fabricate, however this can be achieved within $\sim 5 \%$ and precisely measured. This is followed by cutting the PMQ to the required length for the targeted strength. The individual sources of errors are indirectly incorporated in the quadrupole strength which is included in the presented error analysis. This implies that as long as the targeted strength of the quadrupole is achieved after manufacturing, despite the manufacturing errors, the quadrupole triplet performance can be considered up to the design settings as in simulations. For this reason, manufacturing errors in the quadrupole are not considered individually in the error analysis but are indirectly incorporated in the quadrupole strength. The assumed sources of errors considered are realistic with reasonable tolerances arising from physical positioning setup that can be used to build the quadrupole triplet [52] with ARES capable of delivering the beam within the given variation from the design values. The required tolerances are summarized in Table IV.

There can be other sources of errors critical for the experimental implementation such as errors of the laser beam or the plasma itself. For example the laser could fluctuate in energy, spot size or could have transverse 

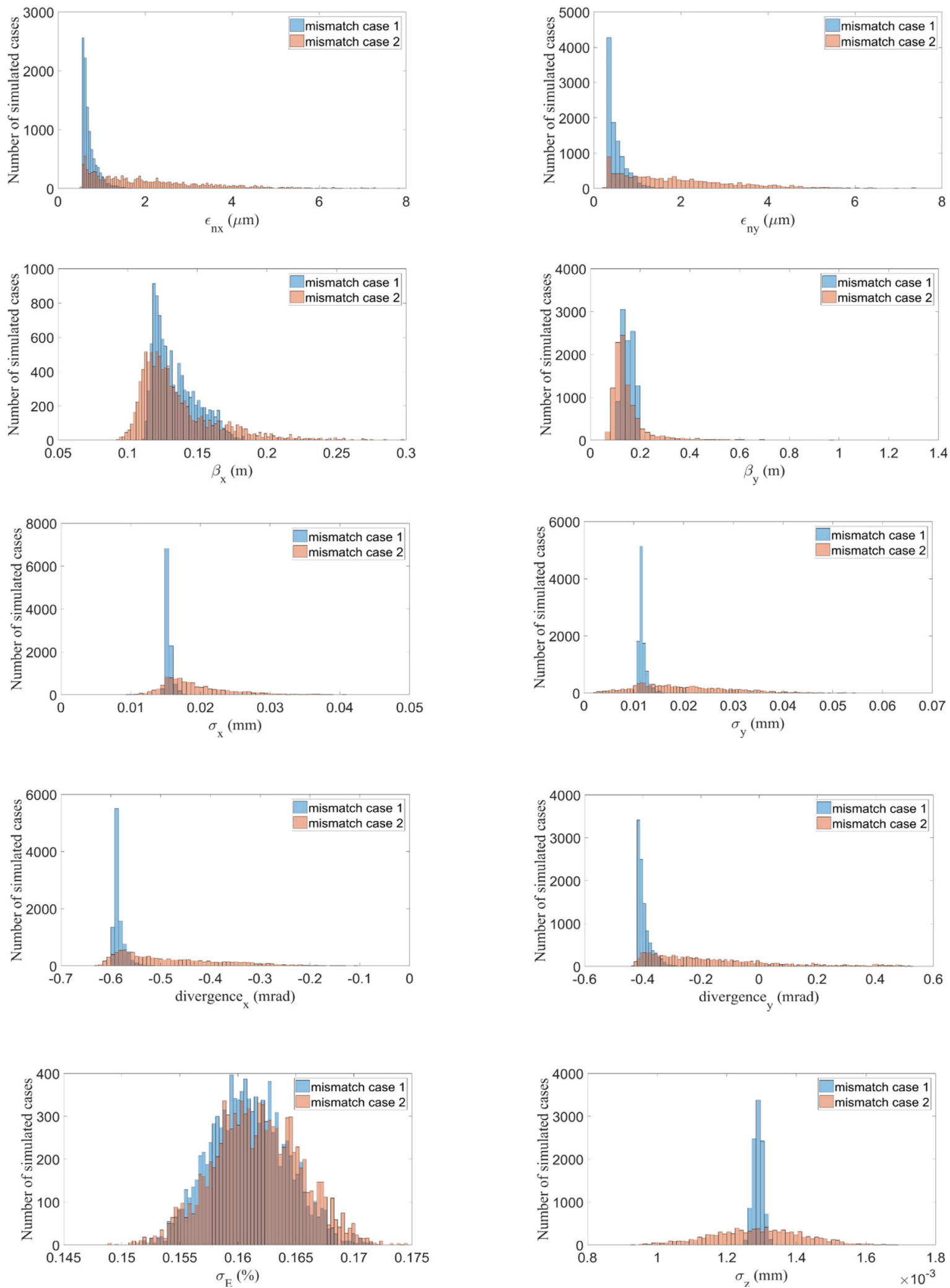

FIG. 7. Variation in observed beam parameters at the plasma entrance for the two error scenarios defined in the text (mismatch cases 1 and 2). 10,000 random cases are simulated for each case. 
TABLE IV. Maximum tolerable variations in the input parameters for the electron beam at the $\mathrm{BC}$ exit, passing through the PMQ triplet and of the PMQ triplet. The shown variations correspond to the case when all other parameters are also varying from their design values within the range.

\begin{tabular}{lcc}
\hline \hline Parameters & Units & Tolerance range \\
\hline Error from the beam & $\mathrm{pC}$ & $10 \%$ \\
Bunch charge & $\mu \mathrm{m}$ & $10 \%$ \\
Transverse beam jitter in x and y & $\mu \mathrm{m}$ & $10 \%$ \\
Bunch size in x and y & $\mathrm{fs}$ & $10 \%$ \\
Bunch length & & \\
Error from the triplet & $\mu \mathrm{m}$ & 10 \\
Transverse misalignment in x and y & $\mu \mathrm{rad}$ & 10 \\
Rotational misalignment of the & & \\
quadrupole in the (x-z), (y-z) & & \\
and (x-y) planes & $\mathrm{mm}$ & $10 \%$ \\
Longitudinal quadrupole position & $\mathrm{mm}$ & $10 \%$ \\
Effective length of the quadrupole & $\mathrm{m}^{-2}$ & $1 \%$ \\
Quadrupole strength &
\end{tabular}

offsets with respect to the plasma cell. Errors from the laser and the plasma source could translate into energy jitters and emittance growth, affecting the performance of the LPA. To quantify the impact of these errors requires a detailed study with a large number of plasma simulations that are computationally expensive. The aim of this paper is to present the design of the final focus system implemented for the LPA beam line, to effectively transport and focus the electron beam to the plasma cell. The simulated acceleration through the plasma, in the next section, validates the design of the final focus system as the transported beam can be efficiently accelerated with usable beam quality after the plasma cell.

\section{BEAM ACCELERATION IN THE LPA}

The LPA stage is designed to boost the energy of the electron beam to $1 \mathrm{GeV}$. It operates at a density of $10^{17} \mathrm{~cm}^{-3}$ and has a symmetric longitudinal density profile, as shown in Fig. 8(a), consisting of a $3 \mathrm{~cm}$ upramp (described in Sec. III), a $15 \mathrm{~cm}$ plateau, and another $3 \mathrm{~cm}$ downramp. In addition, a parabolic transverse density profile $n_{p}(r)=n_{p, 0}+$ $r^{2} / \pi r_{e} w_{0}^{4}$ is used to guide the laser driver, where $r_{e}$ is the classical electron radius and $w_{0}$ is the laser beam size at the focus. Plasma channels able to guide laser pulses with $w_{0} \sim$ $40 \mu \mathrm{m}$ have been experimentally demonstrated [53]. The guiding channel is applied only in the plateau, with the laser pulse being focused at the plateau entrance. The electron beam is injected $50 \mu \mathrm{m}$ behind the laser pulse.

The simulations have been performed with the quasi-3D particle-in-cell code FBPIC [54] using the efficient Lorentzboosted frame method [55]. Two simulations have been carried out, corresponding to a matched case with the ideal beam line design parameters, and a mismatched case, with all the sources of errors in the electron beam and PMQ
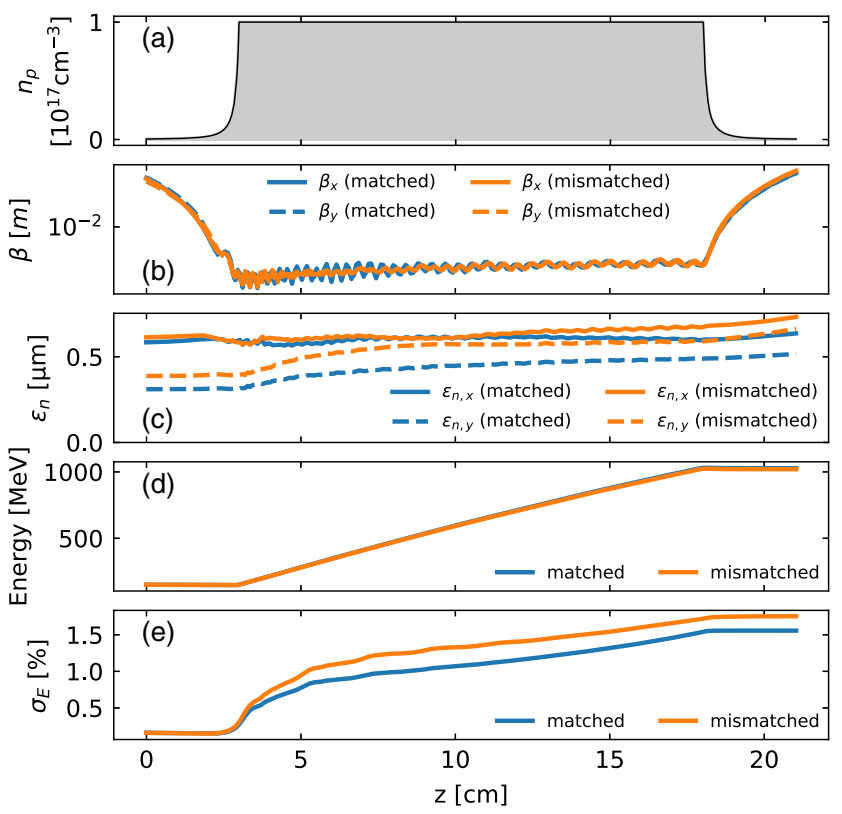

FIG. 8. Simulated evolution of beam parameters through an LPA plasma cell, using FBPIC code, in the ARES linac for the matched beam and the mismatched case 1 beam considered in this study.

triplet parameters introduced within tolerances of mismatch case 1 of Fig. 7. A beam from the mismatch case 2 has not been simulated, since from Fig. 7 the stability does not seem sufficient for reliable injection into the LPA.

The evolution of the beam parameters along the LPA is shown in Fig. 8. Both cases reach a final energy of $1 \mathrm{GeV}$ while exhibiting conservation of the transverse normalized emittance. This demonstrates that the PMQ triplet effectively focuses the beam into the LPA, satisfying the matching criteria from Sec. III. This also shows that the proposed tolerances in the mismatch case 1 could be sufficient for reliable injection into the LPA. The projected rms energy spread grows to a final value of $\sim 1.5 \%$ due to uneven beam loading: while the high-current head manages to partly flatten the accelerating fields, the long and lowcharge tail experiences larger energy gain. By cutting out all tail particles $10 \mathrm{fs}$ or more behind the bunch center (only $1.3 \%$ of the charge), the rms energy spread improves to $0.68 \%$ and $0.83 \%$ in the matched and mismatched cases, respectively, showing that these particles far out in the tail can bias the calculated observables. The average relative slice energy spread is kept at $\sim 0.3 \%$ in both beams. Figure 9 shows the final transverse and longitudinal phase spaces after the LPA, including the $10 \mathrm{fs}$ cut in the tail.

Achieving emittance preservation and a small energy spread is essential for demonstrating the usability of LPA beams. While the emittance is successfully preserved by proper matching with the PMQ triplet, there is still room for improvement with regards to the energy spread, which is significantly larger than current FEL facilities [56]. This 

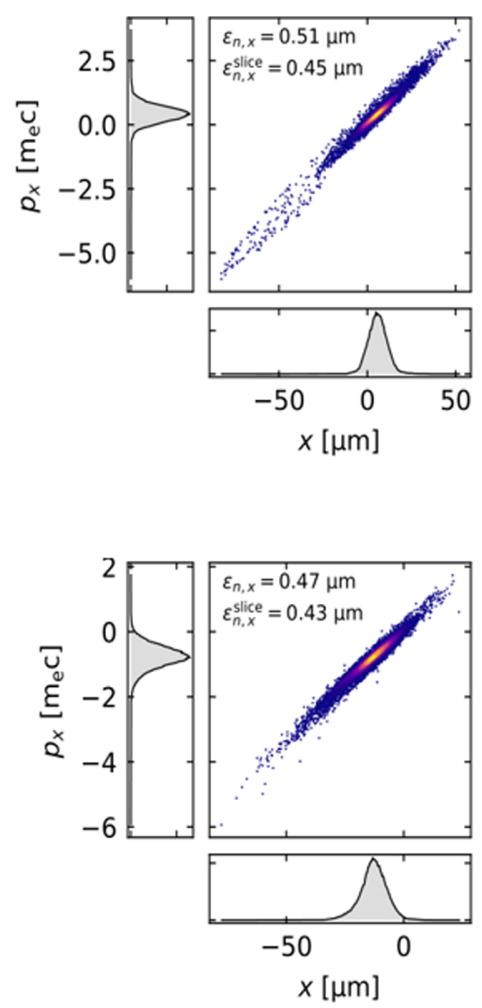

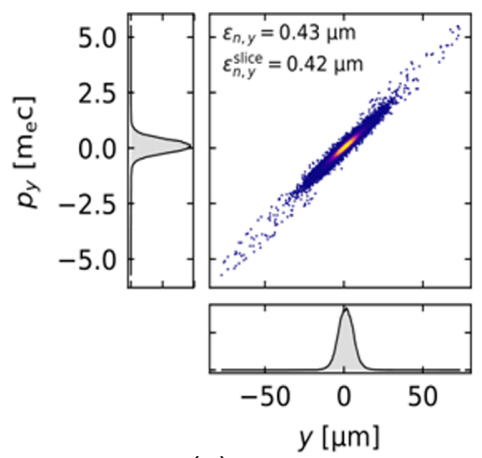

(a)

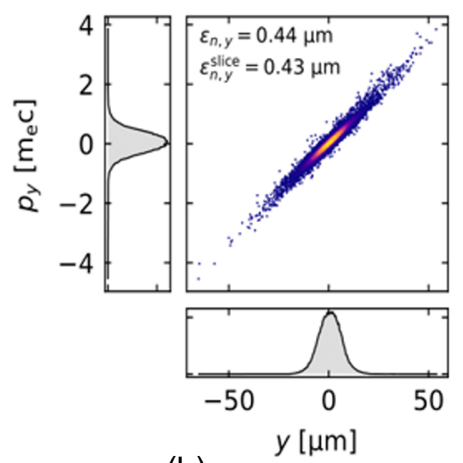

(b)
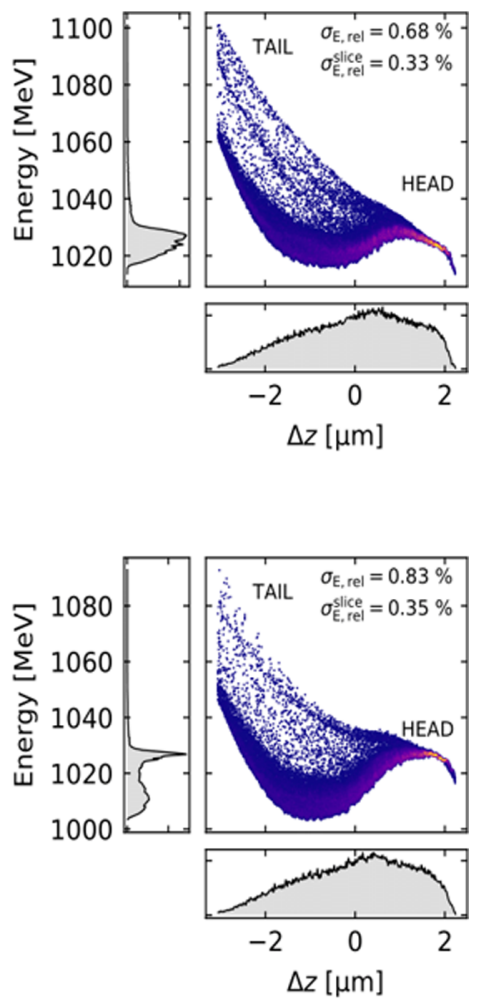

FIG. 9. Phase spaces at the exit of the plasma cell for (a) simulated beam matched to the plasma entrance (reference case from Sec. V) (b) perturbed beam, according to mismatch case 1 of Fig. 7. The beam is tracked through the plasma cell using FBPIC code.

could be achieved by further tailoring the current profile for optimal beamloading. For applications such as FEL, large energy spreads can also be tolerated by reducing the slice energy spread by means of bunch decompression, as demonstrated in [57]. Recently, a scheme has been proposed to diminish the energy spread by using a chicane between two plasma stages [25].

\section{CONCLUSION}

The design for a final focus system, an experimental beam line and an LPA with external injection has been developed for the ARES linac in the SINBAD facility at DESY. Detailed numerical simulations show that the electron beam can be transported and focused to a plasma cell. The studies include the effects of SC. SC plays a significant role and deteriorates beam quality in the beam line after the $\mathrm{BC}$ when beam is compressed to achieve desired bunch length. After the final focus system, the electron beam has adequate transverse symmetry and is well matched into a plasma channel with plasma ramps. The longitudinal phase space is preserved with a $1 \mathrm{kA}$ peak current, as approaching the requirements for several use cases. We have performed a sensitivity analysis of the PMQ triplet for understanding the tolerances and to mitigate the effect of imperfections of the final focus system. The performed error analysis, which could be generalized for any quadrupole triplet, gives a useful estimate about the performance of the final focus system and suggests critical parameters in the implementation of the experiment. The most significant source of error is the quadrupole strength and the longitudinal positioning of the quadrupole triplet as this changes the focusing properties of the triplet.

The main focus of ARES is the generation and characterization of ultrashort pulses and as a test bench for laser based novel external injection acceleration concepts. The beam line is currently under construction. A stepwise commissioning plan will be executed until mid-2024 [7]. Meanwhile the scientific objectives of ARES will be consistently investigated and reviewed. The aim of this article is to present the design of the final focus system which fulfills the matching criterion for the LPA experiment within the defined constraints. The plasma simulations presented validate the design studies. The design constraints are in general typical to any LPA experiment with external injection of electron beam. Matching criterion depends mostly upon the laser and plasma parameters. The design studies for the final focus system and the error analysis presented can be generalized and adopted for any LPA experiment with external injection of electron beam. Other sources of errors that can be of importance for the experiment implementation, such as laser beam or plasma errors, may also exist. The performance of LPA acceleration may be affected by laser and plasma cell errors which 
can cause energy jitter and result in emittance growth. Quantifying the impact of these errors requires an extensive study using a large number of plasma simulations that are computationally expensive to perform. Possible sources of error arising from the electron beam and the final focus system have been studied in detail. The use of a PMQ triplet as a final focus system is a promising scheme which ensures the transport of beam parameters required for LPA within the constraints of experimental beam line. The plasma simulation shows that external injection of short electron bunches into an LPA at ARES can achieve high beam quality and can constitute a stepping stone towards a staged multi-GeV high performance plasma accelerator.

\section{ACKNOWLEDGMENTS}

The authors acknowledge valuable discussions with $\mathrm{K}$. Floettmann, D. Marx, F. Mayet and F. Jafarinia. Special thanks go to W. Leemans for his support and discussion. This research was supported in part through the Maxwell computational resources operated at Deutsches ElektronenSynchrotron DESY, Hamburg, Germany. The authors would also like to acknowledge the FBPIC developers and contributors. The DESY affiliated authors acknowledge support from DESY (Hamburg, Germany), a member of the Helmholtz Association HGF. In addition, we would like to mention our collaboration partners at ATHENA. The Helmholtz Association funds the ATHENA project.

[1] R. Assmann et al., SINBAD_A proposal for a dedicated accelerator research facility at DESY, in Proceedings of the 5th International Particle Accelerator Conference (IPAC'14), Dresden, Germany, 2014, International Particle Accelerator Conference No. 5 (JACoW, Geneva, Switzerland, 2014), pp. 1466-1469.

[2] U. Dorda, B. Marchetti, J. Zhu, F. Mayet, W. Kuropka, T. Vinatier, G. Vaschenko, K. Galaydych, P. A. Walker, D. Marx, R. Brinkmann, R. Assmann, N. H. Matlis, A. Fallahi, and F.X. Kaertner, Status and objectives of the dedicated accelerator R\&D facility SINBAD at DESY, Nucl. Instrum. Methods Phys. Res., Sect. A 909, 239 (2018).

[3] F. Mayet, R. Assmann, J. Bödewadt, R. Brinkmann, U. Dorda, W. Kuropka, C. Lechner, B. Marchetti, and J. Zhu, Simulations and plans for possible DLA experiments at SINBAD, Nucl. Instrum. Methods Phys. Res., Sect. A 909, 213 (2018).

[4] B. Marchetti, R. Assmann, U. Dorda, and J. Zhu, Conceptual and technical design aspects of accelerators for external injection in LWFA, Appl. Sci. 8, 757 (2018).

[5] F.X. Kärtner et al., AXSIS: Exploring the frontiers in attosecond $\mathrm{X}$-ray science, imaging and spectroscopy, Nucl. Instrum. Methods Phys. Res., Sect. A 829, 24 (2016).

[6] E. Panofski, R. W. Assmann, F. Burkart, U. Dorda, K. Floettmann, M. Huening, B. Marchetti, D. Marx, F. Mayet, P. A. Walker, and S. Yamin, Status report of the
SINBAD-ARES rf photoinjector and LINAC commissioning, in Proceedings of the 10th International Particle Accelerator Conference (IPAC'19), Melbourne, Australia, 2019, International Particle Accelerator Conference No. 10 (JACoW Publishing, Geneva, Switzerland, 2019), pp. 906-909.

[7] B. Marchetti et al., SINBAD-ARES-A photoinjector for external injection experiments in novel accelerators at DESY, J. Phys. Conf. Ser. 1596, 012036 (2020).

[8] J. Zhu, R. W. Assmann, M. Dohlus, U. Dorda, and B. Marchetti, Sub-fs electron bunch generation with sub-10-fs bunch arrival-time jitter via bunch slicing in a magnetic chicane, Phys. Rev. Accel. Beams 19, 054401 (2016).

[9] B. Marchetti, R. W. Assmann, U. Dorda, J. Grebenyuk, and J. Zhu, Compression of an electron bunch by means of velocity bunching at ARES, in Proceedings of the International Particle Accelerator Conference (IPAC'15), Richmond, 2015 (JACoW, Geneva, Switzerland, 2015), pp. 1472-1475.

[10] S. Jaster-Merz, R. W. Assmann, F. Burkart, U. Dorda, J. Dreyling-Eschweiler, L. Huth, U. Krämer, and M. Stanitzki, Development of a beam profile monitor based on silicon strip sensors for low-charge electron beams, J. Phys. Conf. Ser. 1596, 012047 (2020).

[11] D. Marx, R. W. Assmann, P. Craievich, K. Floettmann, A. Grudiev, and B. Marchetti, Simulation studies for characterizing ultrashort bunches using novel polarizable X-band transverse deflection structures, Sci. Rep. 9, 19912 (2019).

[12] T. Tajima and J. M. Dawson, Laser Electron Accelerator, Phys. Rev. Lett. 43, 267 (1979).

[13] W. P. Leemans, B. Nagler, A. J. Gonsalves, C. Tóth, K. Nakaruma, C. G. R. Geddes, E. Esarey, C. B. Schroeder, and S. M. Hooker, GeV electron beams from a centimeterscale accelerator, Nat. Phys. 2, 696 (2006).

[14] M. Litos, E. Adli, J. M. Allen, W. An, C. I. Clarke, S. Corde, C. E. Clayton, J. Frederico, S. J. Gessner, S. Z. Green, M. J. Hogan, C. Joshi, W. Lu, K. A. Marsh, W. B. Mori, M. Schmeltz, N. Vafaei-Najafabadi, and V. Yakimenko, $9 \mathrm{GeV}$ energy gain in a beam-driven plasma wakefield accelerator, Plasma Phys. Controlled Fusion 58, 034017 (2016).

[15] A. J. Gonsalves et al., Petawatt Laser Guiding and Electron Beam Acceleration to $8 \mathrm{GeV}$ in a Laser-Heated Capillary Discharge Waveguide, Phys. Rev. Lett. 122, 084801 (2019).

[16] A. R. Maier, N. M. Delbos, T. Eichner, L. Hübner, S. Jalas, L. Jeppe, S. W. Jolly, M. Kirchen, V. Leroux, P. Messner, M. Schnepp, M. Trunk, P. A. Walker, C. Werle, and P. Winkler, Decoding Sources of Energy Variability in a Laser-Plasma Accelerator, Phys. Rev. X 10, 031039 (2020).

[17] J. Grebenyuk, R. Assmann, U. Dorda, and B. Marchetti, Laser-driven acceleration with external injection at SINBAD, in Proceedings of the International Particle Accelerator Conference (IPAC'14), Dresden, Germany, 2014 (JACoW, Geneva, Switzerland, 2014), pp. 15151518.

[18] B. Marchetti et al., Status of the ARES rf gun at SINBAD: From its characterization and installation towards commissioning, in Proceedings of the 9th International 
Particle Accelerator Conference (IPAC'18), Vancouver, BC, Canada, 2018, International Particle Accelerator Conference No. 9 (JACoW Publishing, Geneva, Switzerland, 2018), pp. 1474-1476.

[19] F. Burkart, R. W. Assmann, U. Dorda, J. Hauser, B. Marchetti, W. Kuropka, S. Lederer, F. Lemery, F. Mayet, E. Panofski, and P. Wiesener, The experimental area at the ARES linac, in Proceedings of the 10th International Particle Accelerator Conference (IPAC'19), Melbourne, Australia, 2019 (Ref. [6]), pp. 867-870.

[20] E. N. Svystun, R. W. Assmann, U. Dorda, B. Marchetti, and A. Martinez de la Ossa, Numerical studies on electron beam quality optimization in a laser-driven plasma accelerator with external injection at SINBAD for ATHENAe, in Proceedings of the 10th International Particle Accelerator Conference (IPAC'19), Melbourne, Australia, 2019 (Ref. [6]), pp. 3628-3631.

[21] F. Lemery, R. Assmann, U. Dorda, K. Flottemann, J. Hauser, M. Huning, G. Kube, M. Lantschner, S. Lederer, B. Marchetti, N. Mildner, M. Pelzer, M. Rosan, J. Tiesses, and K. Wittenburg, Overview of the ARES bunch compressor at SINBAD, in Proceedings of the 10th International Particle Accelerator Conference (IPAC'19), Melbourne, Australia (Ref. [6]), pp. 902-905.

[22] W. Leemans, High average power laser plasma accelerator project at DESY, European Advanced Accelerator Conference (2019).

[23] J. Zhu, Design study for generating sub-femtosecond to femtosecond electron bunches for advanced accelerator development at SINBAD, Dissertation, University of Hamburg, 2017.

[24] S. Yamin, R. W. Assmann, U. Dorda, F. Lemery, B. Marchetti, E. Panofski, and P. A. Walker, Design considerations for permanent magnetic quadrupole triplet for matching into laser driven wake field acceleration experiment at SINBAD, J. Phys. Conf. Ser. 1596, 012010 (2020).

[25] A. F. Pousa, A. Martinez de la Ossa, R. Brinkmann, and R. W. Assmann, Compact Multistage Plasma-Based Accelerator Design for Correlated Energy Spread Compensation, Phys. Rev. Lett. 123, 054801 (2019).

[26] J. van Tilborg, S. K. Barber, F. Isono, C. B. Schroeder, E. Esarey, and W. P. Leemans, Free-electron lasers driven by laser plasma accelerators, AIP Conf. Proc. 1812, 020002 (2017).

[27] E. Esarey, C. B. Schroeder, and W. P. Leemans, Physics of laser-driven plasma-based electron accelerators, Rev. Mod. Phys. 81, 1229 (2009).

[28] K. Halbach, Design of permanent multipole magnets with oriented rare earth cobalt material, Nucl. Instrum. Methods 169, 1 (1980).

[29] J. K. Lim, P. Frigola, G. Travish, J. B. Rosenzweig, S. G. Anderson, W. J. Brown, J. S. Jacob, C. L. Robbins, and A. M. Tremaine, Adjustable, short focal length permanentmagnet quadrupole based electron beam final focus system, Phys. Rev. ST Accel. Beams 8, 072401 (2005).

[30] M. P. Anania, E. Brunetti, S. M. Wiggins, D. W. Grant, G. H. Welsh, R. C. Issac, S. Cipiccia, R. P. Shanks, G. G. Manahan, C. Aniculaesei, S. B. van der Geer, M. J. de Loos, M. W. Poole, B. J. A. Shepherd, J. A. Clarke, W. A. Gillespie, A. M. MacLeod, and D. A. Jaroszynski, An ultrashort pulse ultraviolet radiation undulator source driven by a laser plasma wakefield accelerator, Appl. Phys. Lett. 104, 264102 (2014).

[31] G. G. Manahan, E. Brunetti, C. Aniculaesei, M. P. Anania, S. Cipiccia, M. R. Islam, D. W. Grant, A. Subiel, R. P. Shanks, R. C. Issac, G. H. Welsh, S. M. Wiggins, and D. A. Jaroszynski, Characterization of laser-driven single and double electron bunches with a permanent magnet quadrupole triplet and pepper-pot mask, New J. Phys. 16, 103006 (2014).

[32] R. Weingartner, M. Fuchs, A. Popp, S. Raith, S. Becker, S. Chou, M. Heigoldt, K. Khrennikov, J. Wenz, T. Seggebrock, B. Zeitler, Z. Major, J. Osterhoff, F. Krausz, S. Karsch, and F. Grüner, Imaging laser-wakefield-accelerated electrons using miniature magnetic quadrupole lenses, Phys. Rev. ST Accel. Beams 14, 052801 (2011).

[33] M. Fuchs, R. Weingartner, A. Popp, Z. Major, S. Becker, J. Osterhoff, I. Cortrie, B. Zeitler, R. Hörlein, G. D. Tsakiris, U. Schramm, T. P. Rowlands-Rees, S. M. Hooker, D. Habs, F. Krausz, S. Karsch, and F. Grüner, Laser-driven softX-ray undulator source, Nat. Phys. 5, 826 (2009).

[34] G. Travish, P. Frigola, J. Lim, and J. B. Rosenzweig, A pmq-based, ultrashort focal length, final focus system for next generation beam-radiation and beam-plasma experiments, AIP Conf. Proc. 737, 400 (2004).

[35] S. Yamin, R. W. Assmann, U. Dorda, F. Lemery, B. Marchetti, E. Panofski, and P. Walker, Design considerations for permanent magnetic quadrupole triplet for matching into laser driven wakefield acceleration experiment at SINBAD, in Proceedings of the 10th International Particle Accelerator Conference (IPAC'19), Melbourne, Australia (Ref. [6]), pp. 143-146.

[36] H. Wiedemann, Particle Accelerator Physics, Graduate Texts in Physics, 4th ed. (Springer, New York, 2015).

[37] R. Assmann and K. Yokoya, Transverse beam dynamics in plasma based linacs, Nucl. Instrum. Methods Phys. Res., Sect. A 410, 544 (1998).

[38] T. Mehrling, J. Grebenyuk, F. S. Tsung, K. Floettmann, and J. Osterhoff, Transverse emittance growth in staged laserwakefield acceleration, Phys. Rev. ST Accel. Beams 15, 111303 (2012).

[39] K. Floettmann, Adiabatic matching section for plasma accelerated beams, Phys. Rev. ST Accel. Beams 17, 054402 (2014).

[40] I. Dornmair, K. Floettmann, and A. R. Maier, Emittance conservation by tailored focusing profiles in a plasma accelerator, Phys. Rev. ST Accel. Beams 18, 041302 (2015).

[41] X. L. Xu, J. F. Hua, Y. P. Wu, C. J. Zhang, F. Li, Y. Wan, C.-H. Pai, W. Lu, W. An, P. Yu, M. J. Hogan, C. Joshi, and W. B. Mori, Physics of Phase Space Matching for Staging Plasma and Traditional Accelerator Components using Longitudinally Tailored Plasma Profiles, Phys. Rev. Lett. 116, 124801 (2016).

[42] X. Li, A. Chancé, and P. A. P. Nghiem, Preserving emittance by matching out and matching in plasma wakefield acceleration stage, Phys. Rev. Accel. Beams 22, 021304 (2019).

[43] K. V. Lotov, Efficient operating mode of the plasma wakefield accelerator, Phys. Plasmas 12, 053105 (2005). 
[44] A. F. Pousa, R. Assmann, and A. Martinez de la Ossa, WAKE-T: A fast particle tracking code for plasma-based accelerators, J. Phys. Conf. Ser. 1350, 012056 (2019).

[45] K. Floettmann, ASTRA: A space charge tracking algorithm (2011), Vol. 3, p. 2014, https://www.desy.de/ mpyflo/.

[46] M. Borland, ELEGANT: A flexible SDDS-compliant code for accelerator simulation, Argonne National Laboratory Technical Report, Argonne, IL, 2000.

[47] J. Qiang, S. Lidia, R. D. Ryne, and C. Limborg-Deprey, Three-dimensional quasistatic model for high brightness beam dynamics simulation, Phys. Rev. ST Accel. Beams 9 , 044204 (2006).

[48] B. Zeitler, Phase space linearization and external injection of electron bunches into laser-driven plasma wakefields at REGAE, Dissertation, Universität Hamburg, Hamburg, 2017.

[49] S. van der Meer, Improving the power efficiency of the plasma wakefield accelerator, CERN Technical Report Nos. CERN-PS-85-65-AA, CLIC-Note-3, Geneva, 1985.

[50] M. Tzoufras, W. Lu, F. S. Tsung, C. Huang, W. B. Mori, T. Katsouleas, J. Vieira, R. A. Fonseca, and L. O. Silva, Beam Loading in the Nonlinear Regime of Plasma-Based Acceleration, Phys. Rev. Lett. 101, 145002 (2008).
[51] K. V. Lotov, Efficient operating mode of the plasma wakefield accelerator, Phys. Plasmas 12, 053105 (2005).

[52] Smaract hexapods, https://www.smaract.com/positioning_ systems.

[53] R. J. Shalloo, C. Arran, A. Picksley, A. von Boetticher, L. Corner, J. Holloway, G. Hine, J. Jonnerby, H. M. Milchberg, C. Thornton, R. Walczak, and S. M. Hooker, Low-density hydrodynamic optical-field-ionized plasma channels generated with an axicon lens, Phys. Rev. Accel. Beams 22, 041302 (2019).

[54] R. Lehe, M. Kirchen, I. A. Andriyash, B. B. Godfrey, and J.-L. Vay, A spectral, quasicylindrical and dispersion-free particle-in-cell algorithm, Comput. Phys. Commun. 203, 66 (2016).

[55] J.-L. Vay, Noninvariance of Space- and Time-Scale Ranges under a Lorentz Transformation and the Implications for the Study of Relativistic Interactions, Phys. Rev. Lett. 98, 130405 (2007).

[56] Flash accelerator at DESY, https://flash.desy.de/accelerator/.

[57] A. R. Maier, A. Meseck, S. Reiche, C. B. Schroeder, T. Seggebrock, and F. Grüner, Demonstration Scheme for a Laser Plasma Driven Free Electron Laser, Phys. Rev. X 2, 031019 (2012). 